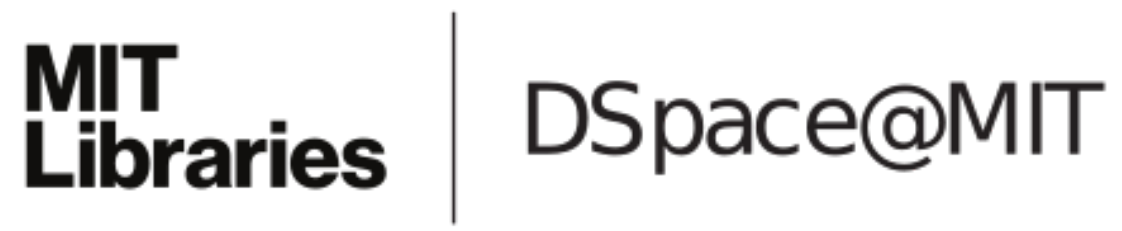

\author{
MIT Open Access Articles
}

Stable arrangements of mobile sensors for sampling physical fields

The MIT Faculty has made this article openly available. Please share how this access benefits you. Your story matters.

Citation: Kumar, Sumeet, Ajay Deshpande and Sanjay E. Sarma. "Stable arrangements of mobile sensors for sampling physical fields." American Control Conference (ACC 2012), 27-29 June 2012, Montreal, QC, p.324-331.

As Published: http://ieeexplore.ieee.org/xpls/abs_all.jsp?arnumber=6315677\&tag=1

Publisher: American Automatic Control Council/IEEE

Persistent URL: http://hdl.handle.net/1721.1/87672

Version: Author's final manuscript: final author's manuscript post peer review, without publisher's formatting or copy editing

Terms of use: Creative Commons Attribution-Noncommercial-Share Alike 


\title{
Stable arrangements of mobile sensors for sampling physical fields
}

\author{
Sumeet Kumar, Ajay Deshpande and Sanjay E. Sarma
}

\begin{abstract}
Today's wireless sensor nodes can be easily attached to mobile platforms such as robots, cars and cell phones enabling pervasive sensing of physical fields (say of temperature, vibrations, air quality and chemicals). We address the sensor arrangement problem, i.e. when and where sensors should take samples to obtain a good estimate of a field using mobile sensors. In particular, we focus on incidentally mobile sensors that move passively under the influence of the environment (e.g. sensors attached to floating buoys, cars and smartphones carried by humans). We model the field as a linear combination of known basis functions. Given the samples, we use a linear estimator to find unknown coefficients of the basis functions. We formulate the sensor arrangement problem as one of finding suitably characterized classes of sensor arrangements that lead to a stable reconstruction of the field. We consider a family of multidimensional $\delta$-dense sensor arrangements, where any square disc of size $\delta$ contains at least one sample, and derive sufficiency conditions for the arrangement to be stable. $\delta$-dense sensor arrangements are geometrically intuitive and are easily compatible with the incidental mobility of sensors in many situations. We present simulation results on the stability of such arrangements for two-dimensional basis functions. We also present an example for constructing basis functions through proper orthogonal decompositions for a one-dimensional chemical diffusion field in a heterogeneous medium, which are later used for field estimation through $\delta$-dense sampling.
\end{abstract}

\section{INTRODUCTION}

W ireless sensor networks (WSN's) have the potential to transform the way we monitor our built and natural environments by providing measurements at spatial and temporal scales that was not possible a few years ago. Today's sensor nodes such as Motes, Sun SPOTS and even smartphones are capable of sensing location, acceleration, light intensity, temperature, pressure, relative humidity, air quality and chemical concentrations using in-built and/or add-on sensors [1], [2]. Thanks to their compact form factors, these nodes can be easily attached to mobile platforms such as robots, cars, buoys, humans and animals to achieve wide-area coverage [3]. For example, air quality sensors attached to taxicabs can map emissions data in urban areas. With such large-scale deployments, sensors could provide unprecedented amounts of data about the

Manuscript received September 25, 2011.

Sumeet Kumar is with the Mechanical Engineering Department, Massachusetts Institute of Technology, Cambridge, MA 02139 USA (phone: 617-324-5121; fax: 617-253-7549; e-mail: sumeetkr@mit.edu).

Ajay Deshpande is with the IBM Research, Hawthorne, NY 10532 and is also a Research Affiliate with the Laboratory for Manufacturing and Productivity, MIT (e-mail: ajayd@mit.edu).

Sanjay E. Sarma is with the Mechanical Engineering Department, Massachusetts Institute of Technology, Cambridge, MA 02139 USA (email: sesarma@mit.edu). environment.

Sensors collect samples of some physical quantity that forms a space-time field over a region of interest. In many cases, we want to reconstruct such a field and use it to predict how it will evolve or know the locations of its hot spots. For example, in the case of chemical spills in a river or an ocean, we want to know the concentration maps and/or the origins of the spill. A fundamental question in this regard is when and where sensors should take samples to obtain a good estimate of the field. We call a geometric configuration of sampling locations (and times) a sensor arrangement and refer to this problem as a sensor arrangement problem. At a high level, a standard approach in the literature to address this problem is to assume some underlying model for the field and find a sensor arrangement that helps to best estimate unknown parameters of the model. Any approach needs to address three issues: sensor noise, modeling error and sensor mobility. Sensor noise stems from errors in measurements whereas modeling error corresponds to the difference between the actual field and the (unknown) model. Previous work has extensively addressed the two issues by developing robust estimation methods to address sensor noise and modeling error. Examples of models studied so far include band-limited functions [4], shiftinvariant functions [4], [5], models based on proper orthogonal decomposition [6], [7], Markov random fields and Gaussian processes [8], [9]. Methods for finding best sensor arrangements search for locations that minimize some error metric associated with the field estimation. Example error metrics include mean squared error [10], weighted least square error [5], variance [11], entropy [8] and mutual information [9].

The third issue in sampling concerns sensor mobility and has become very relevant with recent work in WSN. We view sensor mobility as of two kinds, intentional and incidental [11], [12]. In intentional mobility, a sensor is mounted on a mobile platform that moves to a specified location under its control (e.g. sensors attached to robots and UAVs). In incidental mobility, a sensor is mounted on a platform that moves of its own accord (e.g. sensors attached to cars and smartphones carried by humans). In recent years, researchers have routinely addressed the case of intentionally mobile sensors [13], [14], [15], [16], [17], [18]. However, sampling with incidentally mobile sensors is an emerging issue [11], [12]. In this paper, we address the sensor arrangement problem with a particular focus on incidentally mobile sensors. Unlike intentionally mobile sensors, specifying a particular sensor arrangement for incidentally mobile sensors is of no use, as we do not have 
control over where sensors move. Instead, we take the approach of finding a class of sensor arrangements that are compatible with the mobility of incidentally mobile sensors and lead to a stable reconstruction of the field.

We consider a field that is modeled as a linear combination of known basis functions. In a typical forward parameter estimation problem, a linear estimator finds the estimates of unknown coefficients of the basis functions that minimize the weighted least square error. In the presence of sensor noise and local variations, depending on the sampling locations the linear estimator may be ill-conditioned. We formulate the sensor arrangement problem as one of finding a class of sensor arrangements that lead to well-conditioned linear estimators and guarantee a stable reconstruction of the field. Motivated by the ideas in signal processing literature [5], [19] and [20], we consider a family of $\delta$-dense sensor arrangements in which there is no square hole in the sampling domain of size bigger than $2 \delta$. We derive theoretical conditions under which a function can be reconstructed from $\delta$-dense sampling. Further our results are multidimensional and are thus applicable to a wide range of signals. We find an explicit bound on the condition number of the sampling operator in terms of $\delta$, which only improves with assumptions on the sparsity of the field. $\delta$-dense sensor arrangements are geometrically intuitive and allow for easy encoding of simple sampling rules in incidentally as well as intentionally mobile sensors [11], [12]. We present simulation results on the condition number of direct linear estimators of the field and show that $\delta$-dense sensor arrangements are not only flexible but also robust estimators with small condition numbers. We also derive basis functions for a one-dimensional chemical diffusion field in a heterogeneous medium using proper orthogonal decompositions and present simulation results on the mean squared estimation error in the presence of additive Gaussian noise through $\delta$-dense sensor arrangements.

\section{RELATED WORK}

As discussed earlier, a common approach to addressing the sensor arrangement problem is to assume a model for the underlying field and find a sensor arrangement that yields the best estimate of the field. The sensor arrangement problem is addressed by researchers from different domains including signal processing, computational mechanics, and statistics. In signal processing, one of the classic results in field reconstruction is the Shannon sampling theorem, which states that a band-limited field can be reconstructed using uniformly placed samples at or above the Nyquist rate, i.e. twice the highest frequency of the field [4], [21]. Furthermore uniform arrangements of sensors not only enable faster reconstructions via Fast Fourier Transforms, but are also robust to sensor noise in that they lead to the minimum mean-squared error of reconstruction [22]. In [10], the authors show that there exists a general class of nonuniform sensor arrangements that yield these exact same properties as uniform arrangements. Since Shannon's original result, several researchers have addressed uniform and non-uniform sampling of band-limited and shiftinvariant signal spaces. The non-uniformity of samples does not increase the number of samples but makes the reconstruction problem hard and more sensitive to noise [5], [23], [24]. [5], [19], [20], [23] and [24] consider $\delta$-dense sampling schemes and their stability in the context of trigonometric polynomial fields and shift-invariant fields with applications in image processing and geo-physics. They obtain explicit bounds on $\delta$ for the case of trigonometric polynomials [19], [20]. However they consider only implicit bounds for shift-invariant functions [5]. In this paper, we obtain explicit bounds for general basis functions including proper orthogonal decompositions. In the computational mechanics domain, researchers have considered the optimal sensor placement problem for the reconstruction of convective-diffusive fields modeled using proper orthogonal decompositions [6], [25] and [26]. In statistics, the sensor arrangement problem is the same as the optimal design of experiments [27] and researchers have used the Bayesian inference framework. In most cases, the emphasis has been on finding a particular optimal or near-optimal sensor arrangement for the problem at hand. With recent research in WSN that has enabled pervasive sensing using mobile sensors, non-uniform sampling has become inevitable [28]. Most papers in this domain have addressed the sensor arrangement problem for intentionally mobile sensors (e.g. [13], [14], [15], [16], [17] and [18]) with the exception of [11] and [12]. In [11] and [12] the authors propose a variety of error tolerant sensor arrangements for sampling trigonometric polynomial fields that guarantee that the mean-squared error of reconstruction is less than some error tolerance.

\section{PROBLEM FORMULATION}

In this section, we discuss the formulation of the sensor arrangement problem. We first discuss the parametric modeling of the field in terms of known basis functions and then discuss the linear estimation of the field. For the sake of completeness, we also briefly discuss how to derive basis functions for physical fields using proper orthogonal decompositions.

\section{A. Linear Estimator of the parametric scalar field}

We consider the following parametric model of the unknown scalar field in the $D$ dimensional domain $\vec{x}=\left(x_{1}, \ldots \ldots, x_{D}\right) \in[0,1]^{D} \equiv Q$ :

$f(\vec{x})=\sum_{k=1}^{M} a_{k} \phi_{k}(\vec{x})$,

where $\phi_{k}(\vec{x})$ 's form a set of known $M$ orthonormal basis functions. Orthonormality is defined through the following inner product that we will use in the rest of the paper:

$$
\left\langle\phi_{i}, \phi_{j}\right\rangle=\oint_{Q} \phi_{i}(\vec{x}) \phi_{j}(\vec{x}) d \vec{x}=\delta_{i j},
$$

where $\delta_{i j}$ is the Kronecker delta. $a_{i}$ 's form the set of $M$ unknown coefficients to be estimated. Note that GramSchmidt orthogonalization can be used to create an orthonormal set of basis functions from any initial general 
set of basis functions [29].

Suppose we take samples $y_{i}$ 's of the field at $N \geq M$ distinct sampling locations or sensor locations $\vec{x}_{i} \in[0,1]^{D}$, as shown for a 2D case in Fig. 1. We define $X=\left\{\vec{x}_{i}\right\}_{i=1}^{N}$ as a set of sampling locations (and times) and refer to it as a sensor arrangement. Under the aforementioned setting the field estimation problem boils down to estimating the unknown coefficients $a_{i}$ 's from the data set $\left(\vec{x}_{i}, y_{i}\right)$. We use the following vector and matrix notation:

$$
\begin{aligned}
& \vec{y}=\left(\begin{array}{c}
y_{1} \\
y_{2} \\
\cdot \\
\cdot \\
y_{N}
\end{array}\right)_{N \times 1}, \vec{a}=\left(\begin{array}{c}
a_{1} \\
a_{2} \\
\cdot \\
\cdot \\
a_{M}
\end{array}\right)_{M \times 1},
\end{aligned}
$$

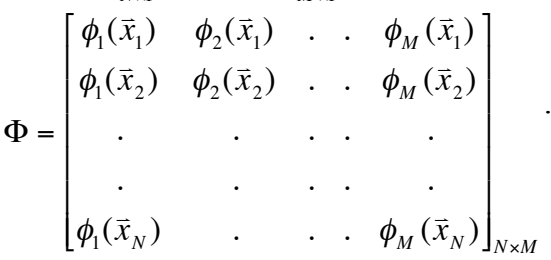

We refer to $\vec{y}$ as an observation vector, $\Phi$ as an observation matrix and $\vec{a}$ as the unknown coefficient vector. In the above setting we have the following observation model:

$\vec{y}=\Phi \vec{a}+\vec{\eta}$,

where $\vec{\eta}$ models additive noise whose origins can be attributed to either sensor noise and/or modeling error.

We use the following weighted least square estimator of $\vec{a}$ which minimizes the weighted residual $\sum_{i=1}^{N} w_{i}\left(f\left(\vec{x}_{i}\right)-y_{i}\right)^{2}$ :

$\overrightarrow{\hat{a}}_{M \times 1}=\left(\Phi^{T} W \Phi\right)_{M \times M}^{-1} \Phi_{M \times N}^{T} W_{N \times N} \vec{y}_{N \times 1}=B^{-1} \Phi^{T} W y$,

where $B=\Phi^{T} W \Phi$ and $w_{i}$ 's form the main diagonal of the weight matrix $W$ [30]. Typically, weights can be chosen in proportion to the separation between points, for example half the nearest neighbor distance of a sampling point [31]. Through the rest of the paper we use this as a choice for our weight matrix. Note that with $w_{i}=1$ we get the regular least square estimator as:

$\overrightarrow{\hat{a}}=B_{2}^{-1} \Phi^{T} \vec{y}$,

where $B_{2}=\Phi^{T} \Phi$. The field can now be reconstructed using:

$\hat{f}(\vec{x})=\sum_{k=1}^{M} \hat{a}_{k} \phi_{k}(\vec{x})$.

\section{B. Sensor Arrangement Problems}

The estimators defined in Eqn. 5 and Eqn. 6 are functions of the sensor arrangement $X$. As discussed before the measurements $y_{i}$ 's can be noisy because of sensor noise and modeling error. An arrangement $X$ is stable if the condition number of the matrix $B$ (or $\left.B_{2}\right), \kappa(B)$ (or $\kappa\left(B_{2}\right)$ ) is close to 1 . This mitigates the effect of noise on the estimate of $\vec{a}$ and leads to higher numerical stability of the estimator defined in
Eqn. 5 (or Eqn. 6). Formally we define the sensor arrangement problem as finding the stable arrangements of $X$.



Fig. 1: A sensor arrangement in 2D with the sampling locations at $\vec{x}_{i}$. Each square partition $S_{i}$ of size $\delta \times \delta$ has at least one sampling location.

\section{Obtaining basis functions and POD's}

Several choices of basis functions such as trigonometric polynomials, band limited functions, shift-invaraint functions have been explored in the literature. As discussed in [6], [7], [25] and [26] the proper orthogonal decomposition (POD) has emerged as a powerful methodology to obtain reduced order models of complex phenomena and have shown success in the domain of fluid modeling, nonlinear dynamics and computational science. In scenarios where the signal is generated by a physical phenomenon governed by a set of differential equations, POD is a promising technique for parametric signal representation through appropriate selection of basis functions. Further, these basis functions need not be bandlimited in the Fourier sense and hence open up new challenges in stable sampling and reconstruction of fields with generalized basis functions.

For the sake of completeness we present a mechanism to derive POD's. Suppose $f(\vec{x}, \mu)$ is a parametric scalar field that is obtained as a result of the solution of a parametric governing differential equation such as chemical diffusion described in section $\mathrm{V}$. The parameter $\mu$ has physical relevance, for example, the position of release of pollutants affecting the diffusion field. As described in [32], we can write the $M$-term approximation as:

$f(\vec{x}, \mu) \approx \sum_{k=1}^{M} \phi_{k}(\vec{x}) a_{k}(\mu)$.

The main question is how to optimally choose $\phi_{k}(\vec{x})$. Although POD's are used for continuous fields, here we 
present its discrete version on a fine resolution grid allowing the use of matrix operations. The function can now be represented as a matrix $A_{P \times Q}$ where each column $a_{q}$ represents $f(\vec{x}, \mu)$ on a fine resolution grid with $P$ points for a particular choice of $\mu$. We can now write the singular value decomposition of $A$ as

$A_{P \times Q}=U_{P \times P} \Lambda_{P \times Q} V_{Q \times Q}^{T}=S V^{T}=\sum_{k=1}^{Q} s_{k} v_{k}^{T}$,

where $U$ comprises of left singular vectors of $\mathrm{A}, \mathrm{V}$ comprises of right singular vectors of $\mathrm{A}, \Lambda$ is the matrix of singular values, $S=U \Lambda, s_{k}$ 's form the columns of $S$ and $v_{k}$ 's the columns of $\mathrm{V}$.

Eqn. 9 is the discrete version of Eqn. 8. Since the singular values are arranged in decreasing order, we can choose the first $M$ POD's $\left(s_{k}\right.$ 's) which are the first $M$ most significant modes as a choice for $\phi_{k}(\vec{x})$ 's on the discrete grid. From the perspective of a real world deployment of sensor network, a prior simulation with a large number of different parameters would help in choosing the most significant basis functions.

\section{IV. $\delta$-DENSE SENSOR ARRANGEMENTS AND STABILITY}

In this section we define the geometry of $\delta$-dense sensor arrangements and derive sufficient conditions for such an arrangement to lead to a stable sampling operator of the generalized basis functions described in section III. For a 2D domain we consider partition of the domain $Q$ into squares $S_{i}$ 's of size $\delta \times \delta$ as shown in Fig. 1. This sensor arrangement can be generalized to higher dimensions by considering partitions of the domain through hypercubes of size $\delta^{D}$ in D dimension. The total number of square partitions $N_{S}=1 / \delta^{2}$. Every square partition $S_{i}$ has at least one sampling location (Fig. 1). We call such a sensor arrangement $X$ as $\delta$-dense. For a $\delta$-dense sensor arrangement, we define the distance of every sampling location to its nearest neighbor as $2 z_{i}$ and define the weight matrix $\mathrm{W}$ as

$$
W=\left[\begin{array}{ccccc}
z_{1} & 0 & \cdot & \cdot & 0 \\
0 & z_{2} & 0 & \cdot & 0 \\
\cdot & \cdot & \cdot & \cdot & \cdot \\
\cdot & \cdot & \cdot & \cdot & \cdot \\
0 & . & . & \cdot & z_{N}
\end{array}\right]_{N \times N} .
$$

We define $V_{\Phi}=\operatorname{span}\left(\left\{\phi_{k}(\vec{x})\right\}_{k=1}^{M}\right)$ as the normed vector space of the orthonormal basis functions $\phi_{k}(\vec{x})$. Note that $V_{\Phi}$ is a finite dimensional vector space. The gradient of the function is defined as $\nabla f(\vec{x})=\left(\frac{\partial f(\vec{x})}{\partial x_{1}}, \frac{\partial f(\vec{x})}{\partial x_{2}}, \ldots ., \frac{\partial f(\vec{x})}{\partial x_{D}}\right)$. We define $A$ as the sampling operator similar to the one defined by Gröchenig [19], [20]. The measurements from a sensor arrangement $X$ can be thought of as an operation $A$ on the function space $f(\vec{x})$. We can write

$$
A f(\vec{x})=P\left(\sum_{i=1}^{N} f\left(\vec{x}_{i}\right) \chi_{i}\right)
$$

where $P$ is the orthogonal projection into $V_{\Phi}$, i.e., $P(f(\vec{x}))=\sum_{k=1}^{M}\left\langle f(\vec{x}), \phi_{k}(\vec{x})\right\rangle \phi_{k}(\vec{x}), \vec{x}_{i}$ 's represent the sampling locations and $\chi_{i}$ 's are the characteristic functions with mutually disjoint support, i.e.

$$
\begin{aligned}
& \begin{aligned}
\chi_{i} & =1 \quad \forall \vec{x} \in S_{i} \\
& =0 \text { o.w. }
\end{aligned} \\
& \text { and } \oint_{Q}\left(\sum_{i=1}^{N_{S}} \chi_{i}\right) d \vec{x}=1 .
\end{aligned}
$$

The following theorems are the main result of this section.

Theorem 1: $\forall f(\vec{x}) \in V_{\Phi}$ and $\mathrm{D} \geq 2$, if $\delta<1 / \sqrt{D \sum_{k=1}^{M} c_{k}^{2}}$ where $c_{k}=\max _{\vec{x} \in Q}\left|\nabla \phi_{k}(\vec{x})\right|$ and there is at least one sampling location in every square (hypercube) partition $S_{i}$, then $X$ is a stable $\delta$-dense sensor arrangement and Eqn. 5 or Eqn. 6 provides stable parameter estimation.

For the special case of one-dimensional signals (Fig. 2) we have the following result:

Theorem 2: For $\mathrm{D}=1, \forall f\left(x_{1}\right) \in V_{\Phi}$, if $\delta<\pi /\left(2 \sqrt{\sum_{k=1}^{M} b_{k}^{2}}\right)$ where $b_{k}^{2}=\int_{0}^{1}\left(\frac{d \phi_{k}\left(x_{1}\right)}{d x_{1}}\right)^{2} d x_{1}$ and there is at least one sampling location in every partition $S_{i}$, then $X$ is a stable $\delta$-dense sensor arrangement and Eqn. 5 or Eqn. 6 provides stable parameter estimation.

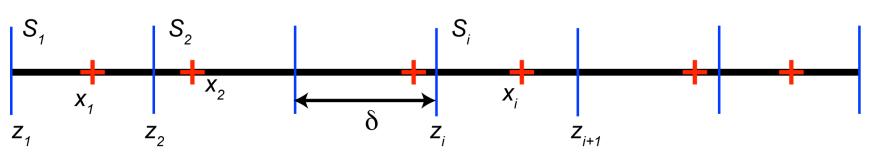

Fig. 2: A sensor arrangement in $1 \mathrm{D}$ with the sampling locations at $x_{i}$ and partitions of size $\delta$ denoted by $S_{i}$.

In order to prove the theorem, we make use of the following lemmas.

Lemma 1: Described in [19], [20].

Let $A$ be a bounded operator on a Banach space $B$ and if $\|f(\vec{x})-A f(\vec{x})\| \leq \gamma\|f(\vec{x})\| \quad \forall \quad f(\vec{x}) \in B$ and $0 \leq \gamma<1$ then $A$ is invertible and is a stable sampling operator for $f(\vec{x})$.

Lemma 2: Using Cauchy-Schwarz inequality the following relationship holds $\forall \alpha_{k}, \beta_{\boldsymbol{k}} \in R$

$\left(\sum_{k=1}^{M} \alpha_{k} \beta_{k}\right)^{2} \leq \sum_{k=1}^{M} \alpha_{k}^{2} \sum_{k=1}^{M} \beta_{k}^{2}$.

Lemma 3: Wirtinger's inequality described in [19], [20].

If $f\left(x_{1}\right), \quad f^{\prime}\left(x_{1}\right)=\frac{d f\left(x_{1}\right)}{d x_{1}} \in L^{2}(a, b)$ and either $f(a)=0$ or $f(b)=0$, then

$\int_{a}^{b}\left|f\left(x_{1}\right)\right|^{2} d x_{1} \leq \frac{4}{\pi^{2}}(b-a)^{2} \int_{a}^{b}\left|f^{\prime}\left(x_{1}\right)\right|^{2} d x_{1}$. 
Proof of Theorem 1: For a $\delta$-dense sensor arrangement $X$, we have

$$
\begin{aligned}
& \|f(\vec{x})-A f(\vec{x})\|^{2}=\left\|f(\vec{x})-P\left(\sum_{i=1}^{N} f\left(\vec{x}_{i}\right) \chi_{i}\right)\right\|^{2} \\
& =\left\|P\left(\sum_{i=1}^{N}\left(f(\vec{x})-f\left(\vec{x}_{i}\right)\right) \chi_{i}\right)\right\|^{2} \\
& \leq\left\|\sum_{i=1}^{N}\left(f(\vec{x})-f\left(\vec{x}_{i}\right)\right) \chi_{i}\right\|^{2} \\
& \leq \sum_{i=1}^{N}\left\|\left(f(\vec{x})-f\left(\vec{x}_{i}\right)\right) \chi_{i}\right\|^{2} \\
& =\sum_{i=1}^{N} \oint\left|f(\vec{x})-f\left(\vec{x}_{i}\right)\right|^{2} \chi_{i} d \vec{x} .
\end{aligned}
$$

Now, for a convex domain $S_{i}$ and using multivariate mean value theorem we can write

$f(\vec{x})-f\left(\vec{x}_{i}\right)=\left(\vec{x}-\vec{x}_{i}\right) \cdot \nabla f(\vec{c})$,

$\forall \vec{x} \in S_{i}$ and for some $\vec{c}$ on the line connecting $\vec{x}$ and $\vec{x}_{i}$. Since $S_{i}$ is a convex domain, we further have $\left|f(\vec{x})-f\left(\vec{x}_{i}\right)\right| \leq\left|\left(\vec{x}-\vec{x}_{i}\right)\right||\nabla f(\vec{c})|, \forall \vec{c} \in S_{i}$

where $|\vec{x}|=\sqrt{\sum_{j=1}^{D} x_{j}^{2}}$.

Taking the maximum of the right hand side gives

$\left|f(\vec{x})-f\left(\vec{x}_{i}\right)\right| \leq \max _{\vec{x} \in S_{i}}\left|\left(\vec{x}-\vec{x}_{i}\right)\right| \max _{\vec{x} \in S_{i}}|\nabla f(\vec{x})|$.

Due to the geometry of $\delta$-dense sensor arrangement the maximum distance between 2 points in a $D$ dimensional hypercube of size $\delta^{D}$ is $\sqrt{D} \delta$. Also using Cauchy-Schwarz inequality we get:

$|\nabla f(\vec{x})| \leq \sqrt{\sum_{k=1}^{M} a_{k}^{2}} \sqrt{\sum_{k=1}^{M}\left|\nabla \phi_{k}(\vec{x})\right|^{2}}$.

Hence we can write, $\forall \vec{x} \in S_{i}$

$$
\begin{aligned}
& \left|f(\vec{x})-f\left(\vec{x}_{i}\right)\right| \leq \sqrt{D} \delta \sqrt{\sum_{k=1}^{M} a_{k}^{2}} \sqrt{\sum_{k=1}^{M} \max _{\vec{x} \in Q}\left|\nabla \phi_{k}(\vec{x})\right|^{2}} \\
& \Rightarrow\left|f(\vec{x})-f\left(\vec{x}_{i}\right)\right| \leq \sqrt{D} \delta \sqrt{\sum_{k=1}^{M} a_{k}^{2}} \sqrt{\sum_{k=1}^{M} c_{k}^{2}} .
\end{aligned}
$$

Since $f(\vec{x}) \in V_{\Phi},\|f(\vec{x})\|^{2}=\sum_{k=1}^{M} a_{k}^{2}$. Using Eqn. 15, Eqn. 21, $N=N_{S}$, the fact that $\chi_{i}$ 's have disjoint support and Eqn. 12 we can write

$$
\begin{aligned}
& \|f(x)-A f(x)\|^{2} \leq \delta^{2} D\left(\sum_{k=1}^{M} c_{k}^{2}\right)\left(\sum_{k=1}^{M} a_{k}^{2}\right) \oint_{Q}\left(\sum_{i=1}^{N} \chi_{i}\right) d \vec{x}, \\
& =\delta^{2} D\left(\sum_{k=1}^{M} c_{k}^{2}\right)\left(\sum_{k=1}^{M} a_{k}^{2}\right)=\delta^{2} D\left(\sum_{k=1}^{M} c_{k}^{2}\right)\|f(\vec{x})\|^{2} .
\end{aligned}
$$

Now using Lemma 1, the sampling operator which is dependent on the sampling arrangement is stable for $\delta<1 / \sqrt{D \sum_{k=1}^{M} c_{k}^{2}}$. Hence $\delta^{*}=1 / \sqrt{D \sum_{k=1}^{M} c_{k}^{2}}$ is the critical value of $\delta$ below which any $\delta$-dense sensor arrangement is stable.
Proof of Theorem 2: For a $\delta$-dense sensor arrangement $X$ in $1 \mathrm{D}$ (Fig. 2), we have

$$
\begin{aligned}
& \left\|f\left(x_{1}\right)-A f\left(x_{1}\right)\right\|^{2}=\left\|f\left(x_{1}\right)-P\left(\sum_{i=1}^{N} f\left(x_{1_{i}}\right) \chi_{i}\right)\right\|^{2} \\
& =\left\|P\left(\sum_{i=1}^{N}\left(f\left(x_{1}\right)-f\left(x_{1 i}\right)\right) \chi_{i}\right)\right\|^{2} \\
& \leq\left\|\sum_{i=1}^{N}\left(f\left(x_{1}\right)-f\left(x_{1 i}\right)\right) \chi_{i}\right\|^{2} \\
& =\int_{0}^{1}\left(\sum_{i=1}^{N}\left(f\left(x_{1}\right)-f\left(x_{1_{i}}\right)\right) \chi_{i}\right)^{2} d x \\
& =\sum_{i=1}^{N} \int_{z_{i}}^{z_{i+1}}\left(f\left(x_{1}\right)-f\left(x_{1_{i}}\right)\right)^{2} d x .
\end{aligned}
$$

Now we can write

$$
\begin{aligned}
& \int_{z_{i}}^{z_{i+1}}\left(f\left(x_{1}\right)-f\left(x_{1_{i}}\right)\right)^{2} d x \\
& =\int_{z_{i}}^{x_{i}}\left(f\left(x_{1}\right)-f\left(x_{1_{i}}\right)\right)^{2} d x+\int_{x_{i}}^{z_{i+1}}\left(f\left(x_{1}\right)-f\left(x_{1_{i}}\right)\right)^{2} d x .
\end{aligned}
$$

Using Lemma 3

$\int_{z_{i}}^{x_{i}}\left(f\left(x_{1}\right)-f\left(x_{1 i}\right)\right)^{2} d x+\int_{x_{i}}^{z_{i+1}}\left(f\left(x_{1}\right)-f\left(x_{1 i}\right)\right)^{2} d x$

$\leq \frac{4 \delta^{2}}{\pi^{2}}\left(\int_{z_{i}}^{z_{i+1}}\left(f^{\prime}\left(x_{1}\right)\right)^{2} d x\right)$

Using Eqn. 23 and 25

$\left\|f\left(x_{1}\right)-A f\left(x_{1}\right)\right\|^{2} \leq \frac{4 \delta^{2}}{\pi^{2}}\left(\int_{0}^{1}\left(f^{\prime}\left(x_{1}\right)\right)^{2} d x\right)$.

Now, $f^{\prime}\left(x_{1}\right)=\sum_{k=1}^{M} a_{k} \phi_{k}^{\prime}\left(x_{1}\right), b_{k}^{2}=\int_{0}^{1}\left(\frac{d \phi_{k}\left(x_{1}\right)}{d x_{1}}\right)^{2} d x_{1}$ and using

Cauchy-Schwarz inequality, we have

$$
\begin{aligned}
& \left\|f^{\prime}(x)\right\|^{2} \leq \sum_{k=1}^{M} \sum_{l=1}^{M} a_{k} a_{l} b_{k} b_{l}=\left(\sum_{k=1}^{M} a_{k} b_{k}\right)^{2} \\
& \Rightarrow\|f(x)-A f(x)\|^{2} \leq \frac{4 \delta^{2}}{\pi^{2}}\left(\sum_{k=1}^{M} a_{k} b_{k}\right)^{2}
\end{aligned}
$$

Now using Lemma 2 we can write

$\left\|f\left(x_{1}\right)-A f\left(x_{1}\right)\right\|^{2} \leq \frac{4 \delta^{2}}{\pi^{2}}\left(\sum_{k=1}^{M} b_{k}^{2}\right)\left(\sum_{k=1}^{M} a_{k}^{2}\right)=\frac{4 \delta^{2}}{\pi^{2}}\left(\sum_{k=1}^{M} b_{k}^{2}\right)\left\|f\left(x_{1}\right)\right\|^{2}$.

Now using Lemma 1, the sampling operator for $1 \mathrm{D}$ signals is stable for $\delta<\pi / 2 \sqrt{\sum_{k=1}^{M} b_{k}^{2}}$. Hence $\delta^{*}=\pi / 2 \sqrt{\sum_{k=1}^{M} b_{k}^{2}}$ is the critical value of $\delta$ below which any $\delta$-dense sensor arrangement is stable.

Note that the bounds in Eqn. 22 and Eqn. 28 present a worst-case analysis. In many cases we may have few dominating coefficient $a_{k}$ 's and hence the bound will be much lower. For example, if the basis function with the maximum $c_{k}$ (for 1D: $b_{k}$ ) has very low $a_{k}$ or in some cases we may have sparse fields or few dominating coefficients $a_{k}$ the resulting bound will be lower. Nonetheless the 
theoretical bounds provide insights into the factors controlling reconstruction of signals through $\delta$-dense sampling and a good starting point to select $\delta$.

Further the operator norm which is defined as $\|A\|_{o p}=\|A f(\vec{x})\| /\|f(\vec{x})\|, \quad$ has the following range $1-\delta \sqrt{D \sum_{k=1}^{M} c_{k}^{2}} \leq\|A\|_{o p} \leq 1+\delta \sqrt{D \sum_{k=1}^{M} c_{k}^{2}}$

$$
1-\frac{2 \delta \sqrt{\sum_{k=1}^{M} b_{k}^{2}}}{\pi} \leq\|A\|_{o p} \leq 1+\frac{2 \delta \sqrt{\sum_{k=1}^{M} b_{k}^{2}}}{\pi} \text { (1D signals). }
$$

Let $\kappa(A)$ denote the upper bound on the condition number of the operator $A$. For $\mathrm{D} \geq 2$ and using Eq. 29 we have:

$\kappa(A)=\left(1+\delta \sqrt{D \sum_{k=1}^{M} c_{k}^{2}}\right) /\left(1-\delta \sqrt{D \sum_{k=1}^{M} c_{k}^{2}}\right)$.

Since we are considering the sensing model as described in Eqn. 1 and a finite dimensional vector space of functions $V_{\Phi}$, estimating the unknown coefficient vector is sufficient for reconstructing the scalar field. We claim that the conditions derived above are sufficient conditions leading to stable sampling and parameter estimation in the presence of noise. Obtaining parameters involve inverting the sampling operator that can be achieved either directly [11] or an iterative approach [19]. Here we consider a direct approach using the weighted least square estimator (Eqn. 5) and least square estimator (Eqn. 6). The condition numbers $\kappa(B)$ and $\kappa\left(B_{2}\right)$ of the matrices $B=\Phi^{T} W \Phi$ and $B_{2}=\Phi^{T} \Phi$ respectively controls the stability of the estimators. High condition numbers amplify the effect of noise on the parameter estimation.

For a sensor arrangement requiring sampling at specific locations mobile sensing platforms would need accurate and sophisticated position controllers making it expensive and energy inefficient. On the other hand $\delta$-dense arrangements open up the possibility of using simpler, energy efficient position controllers for mobile sampling. The only constraint that the $\delta$-dense arrangements impose is that every square partition of size $\delta$ has one sample in it. For instance, in case of sampling air quality using sensors mounted on taxicabs, $\delta$-dense sensor arrangement simply means that we need to take a sample once at least in a span of $\delta$ distance. Further, while dropping sensors from UAV's, we just need to make sure that it leads to a $\delta$-dense arrangement.

\section{NUMERICAL SIMULATIONS}

In this section we present simulation results on the stability of linear estimators and field reconstruction error in the presence of noise.

\section{A. Stability of linear estimators in $\delta$-dense arrangements}

We now present the stability (condition number) of the linear estimator discussed in section III-A. We consider two classes of basis functions in 2D, polynomial and cosine basis functions.

Consider the following set of basis functions: $\varphi_{k}\left(x_{1}, x_{2}\right)=x_{1}^{p} x_{2}^{q}$,

where $p=q=\{0,1,2,3\}$, leading to 16 basis functions in the set. We use the aforementioned set to create 16 orthonormal basis functions $\phi_{k}$ 's in two dimensions using Gram-Schmidt orthogonalization [29]. We follow the same procedure with cosine basis functions:

$$
\varphi_{k}\left(x_{1}, x_{2}\right)=\cos \left(p x_{1}\right) \cos \left(q x_{2}\right) \text {, }
$$

to create another set of orthonormal basis functions.

For a given $\delta$ we simulate 1000 randomly generated $\delta$ dense arrangements (Section IV) and calculate the mean and the standard deviation of $\kappa(B)$ and $\kappa\left(B_{2}\right)$. Fig. 3 shows the variation of the mean condition numbers with $\delta$ for the two sets of basis functions. The error bars indicate the corresponding $1 / 2$ standard deviations. For polynomial basis functions $\delta^{*}=0.0036$ and for cosine basis functions $\delta^{*}=$ 0.0014 . We observe that the theoretical bounds are very conservative and numerically we find that $\delta \leq 0.025$ gives the mean condition number $\leq 1.4$ with standard deviation $\leq$ 0.15 for both polynomial and cosine basis functions. Further we observe that $\kappa\left(B_{2}\right) \leq \kappa(B)$ implying that in the context of $\delta$-dense arrangements generated using our method, the least square estimator is more robust to additive noise as compared to the weighted least square estimator. Though $\delta$ dense arrangements are probabilistic in nature, the mean condition numbers are small with acceptable variance for low values of $\delta$, providing a suitable framework for sampling parametric signals.
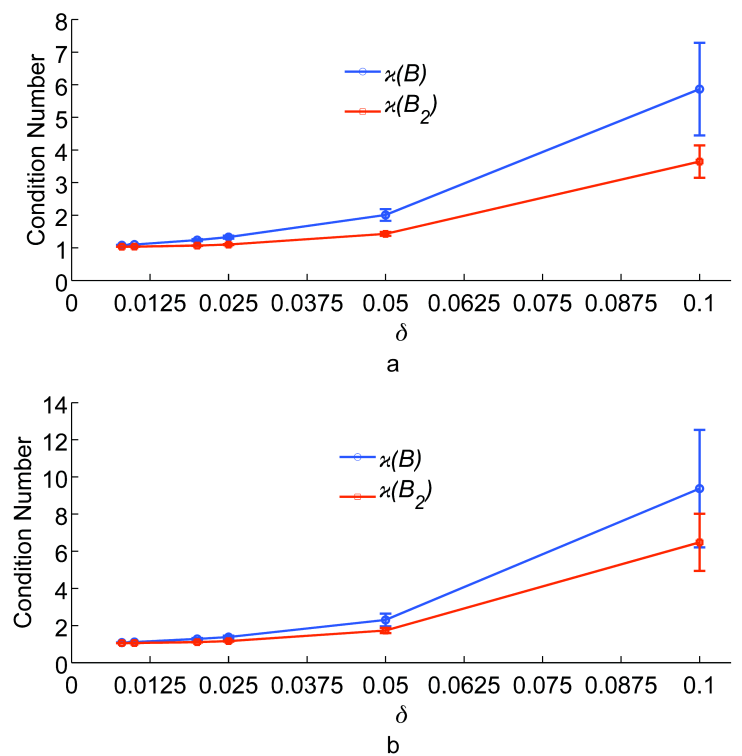

Fig. 3: Variation of the mean of condition numbers of $B$ and $B_{2}$ with $\delta$ for 2D a) orthonormal polynomial basis functions; b) orthonormal cosine basis functions. For $\delta \leq 0.025$ we obtain small condition numbers implying higher numerical stability and robustness to noise. The error bars indicate $1 / 2$ standard deviations.

\section{B. Generating POD's and error in field reconstruction}

We now consider an example of constructing basis functions through POD (Section III-C). Consider the following one-dimensional model of unsteady chemical diffusion in a heterogeneous medium with a source term [33]: 
$\frac{d C\left(x_{1}, t\right)}{d t}=\frac{d}{d x_{1}}\left(D\left(x_{1}\right) \frac{d C\left(x_{1}, t\right)}{d x_{1}}\right)+S(\mu)$,

where $C\left(x_{1}, t\right)$ is the chemical concentration field, $D\left(x_{1}\right)$ is the diffusion coefficient and $S(\mu)$ is the source term.

We consider the field at a particular time instant $T_{o}$, when it changes very slowly in time and is close to the steady state. Such steady chemical fields typically model constant release of gas or oil in a quiescent environment, like oil leak from underwater pipes in a lake. We further assume that the source term is a narrow Gaussian impulse of known amplitude and variance but of unknown mean position $\mu \in$ $[0,1]$, which acts for all times. The particular choice of the source term models the release of chemicals at an unknown location in the domain. We first generate the POD's according to the theory described in Section III-C. We carry out simulation with different values of $\mu$ between 0 and 1 in increments of 0.01 and use the resulting fields to generate the POD's. Fig. 4a shows the variation of $D\left(x_{1}\right)$, an example source term $S(\mu)$ and the resulting concentration field $C\left(x_{1}, T_{o}\right)$ for $\mu=0.45$. The particular profile of $D\left(x_{1}\right)$ was chosen to model a domain where certain regions have higher diffusion coefficients leading to faster diffusion of chemical species. First 10 POD's were chosen as the model basis functions for $C\left(x_{I}\right)$. For the sake of illustration, we show the first 5 POD's with the corresponding singular values in Fig. $4 b$.
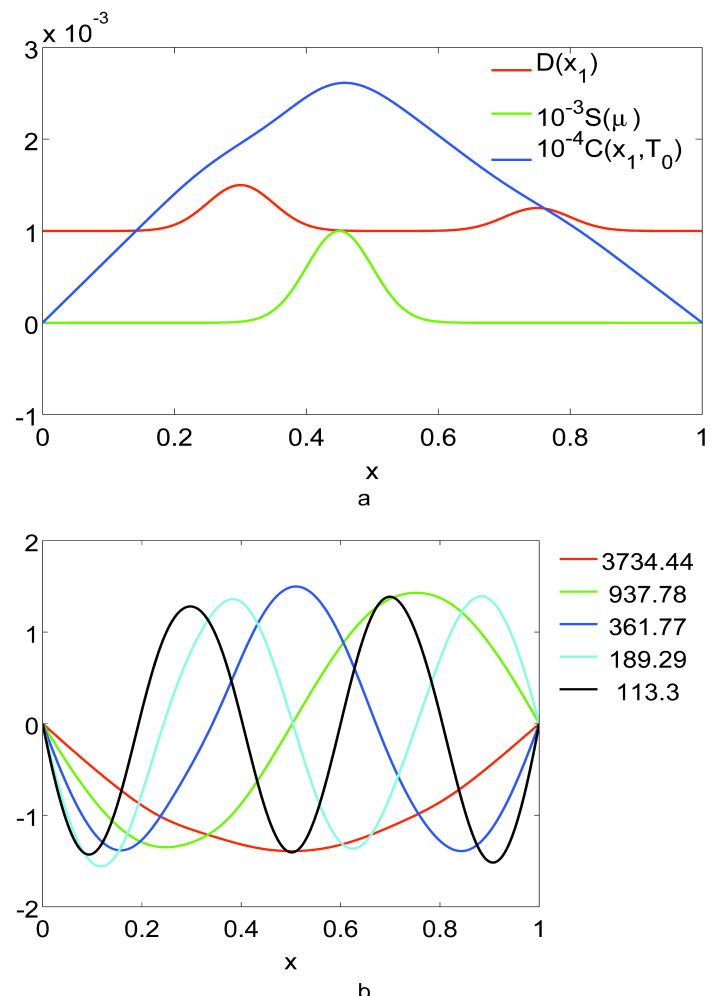

Fig. 4: a) Variation of diffusivity, source term and chemical concentration profile in the domain; b) First 5 POD basis functions and the corresponding singular values generated for Eqn. 34.

We evaluate the performance of $\delta$-dense sensor arrangements in reconstructing $C\left(x_{1}, T_{0}\right)$ for a particular value of $\mu=0.455$ different from the ones used for learning the POD basis functions. Gaussian noise, $\eta=N\left(0, \alpha \times \max \left|C\left(x_{1}, T_{o}\right)\right|\right)$ was added to the measurement vector $\vec{y}$ for each choice of $\alpha$ from the set $\{0.01,0.03,0.05\}$ with $\max \left|C\left(x_{1}, T_{o}\right)\right|=26.1996$ and $\left\|C\left(x_{1}, T_{o}\right)\right\|=16.3665$. Using our method described in Section IV we find $\delta^{*}=$ 0.0252 for POD basis functions. We perform simulations with different values of $\delta$ and for each $\delta$ we generate 1000 arrangements to compute the mean and the standard deviation of condition numbers and the mean squared estimation errors (Fig 5a,b,c). Similar to Fig. 3, we observe that for low $\delta$ both the mean condition numbers are small with reasonable variance. Further the mean squared error in the field estimation is $\leq 7.5 \%$ even at high levels of noise ( $\alpha$ $=0.05)$ for $\delta \leq 0.5$.
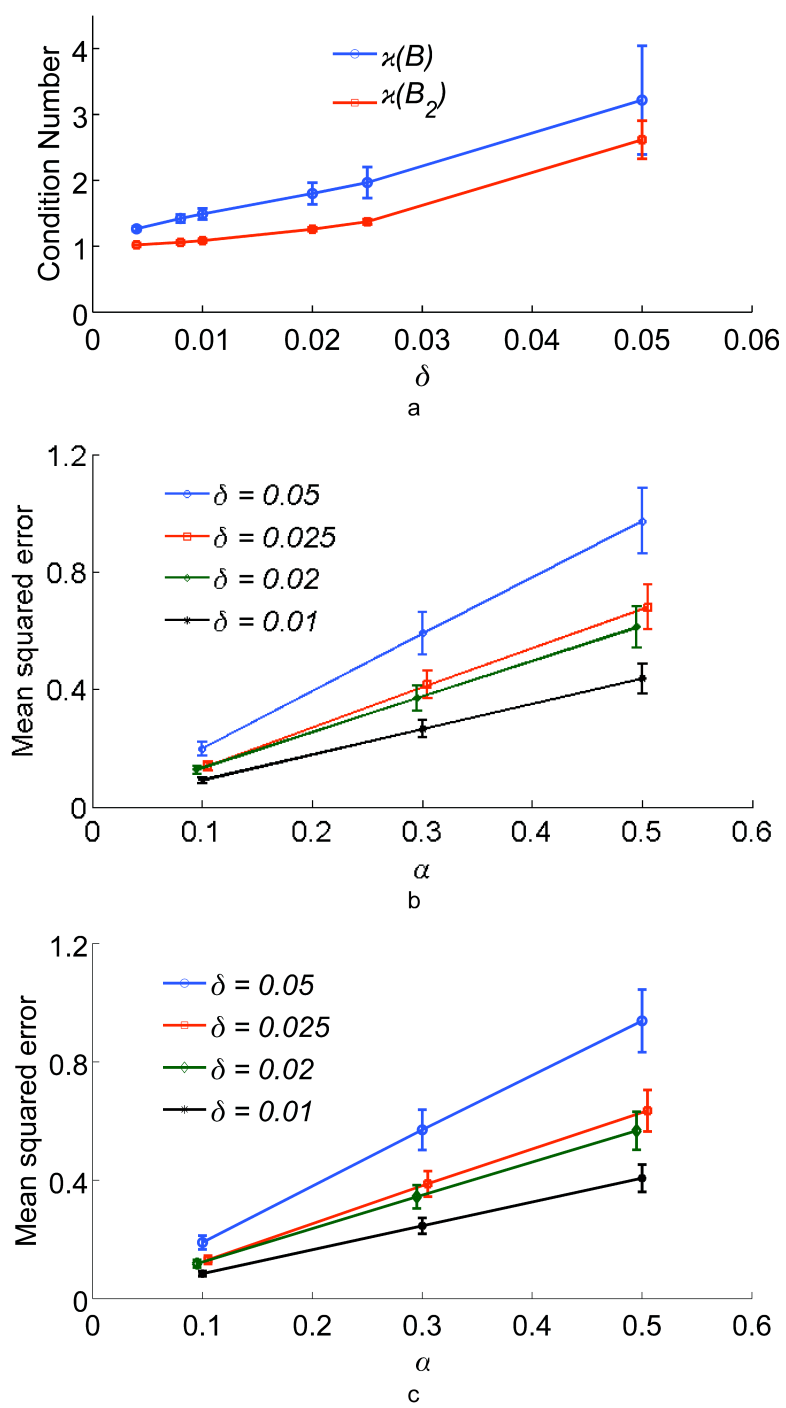

Fig. 5: a) Variation of the mean of condition numbers of $B$ and $B_{2}$ with $\delta$ for 1D POD's; b,c) Mean squared estimation error in reconstructing test function through $\delta$-dense sampling with different magnitudes of additive noise. We report error in direct reconstruction through b) weighted least squares (Eqn. 5) and c) least squares (Eqn. 6). The error bars indicate 1/2 standard deviations. 


\section{CONCLUSION AND FUTURE WORK}

We addressed the sensor arrangement problem - when and where sensors should take samples to obtain a stable reconstruction of a physical field. We modeled the field as a linear combination of known basis functions and used linear estimators for the reconstruction of the field. We considered a family of $\delta$-dense sensor arrangements and obtained sufficient conditions $\left(\delta<\delta^{*}\right)$ for $\delta$-dense arrangements to lead to a stable estimator. Our results are multidimensional making the $\delta$-dense sampling framework suitable for a broad class of signals. The bound on $\delta$ only improves with the field sparsity assumptions. Using numerical simulations, we showed that $\delta$-dense arrangements yield stable reconstructions of the field in the presence of sensor noise and modeling error. We also noted that $\delta$-dense arrangements yield sufficient flexibility in terms of sampling using incidentally mobile sensors. In our future work, we would like to explore the field sparsity further and improve bounds on $\delta$. We would also like to incorporate the effect of sample location error in the sampling framework. In addition, we would like to consider explicitly time varying dynamic fields and explore classes of stable adaptive sensor arrangements.

\section{ACKNOWLEDGMENT}

The authors acknowledge members of the Field Intelligence Laboratory, MIT and Sriram Krishnan for useful discussions and comments on the manuscript.

\section{REFERENCES}

[1] I. F. Akyildiz, W. Su, Y. Sankarasubramaniam and E. Cayirci, "A survey on Sensor Networks," IEEE Communications Magazine, vol. 40, no. 8, pp. 102-114, Aug. 2002.

[2] D. Culler, D. Estrin and M. Srivastave, "Overview of sensor networks," Computer, vol. 37, no. 8, pp. 41-49, Aug. 2004.

[3] P. Dutta, P.M. Aoki, N. Kumar, A. Mainwaring, C. Myers, W. Willett and A. Woodruff, "Demo abstract - Common Sense: Participatory Urban Sensing Using a Network of Handheld Air Quality Monitors," in Proc. 7th ACM Conf. on Embedded Networked Sensor Systems (SenSys '09), pp. 349-350, Nov. 2009.

[4] M. Unser, "Sampling_50 years after Shannon," Proc. IEEE, vol. 88, no. 4, pp. 569-587, Apr. 2000.

[5] A. Aldroubi and K. Gröchenig, "Nonuniform sampling and reconstruction in shift-invariant spaces," SIAM Rev., vol. 43, no. 4, pp. 585-620, 2001

[6] N. C. Nguyen, A. T. Patera and J. Peraire, “A 'best points' interpolation method for efficient approximation of parametrized functions," Int J Numer Methods Eng, vol. 73, pp. 521-543, 2008.

[7] C. W. Rowley, T. Colonius and R. M. Murray, "Model reduction for compressible flows using POD and Galerkin projection," Physica DNonlinear Phenomena, vol. 189, pp. 115-129, 2004.

[8] T. Jaakkola and M. Collins, "6.867 machine learning class notes, MIT,” http://courses.csail.mit.edu/6.867/syllabus.html

[9] A. Krause, A. Singh and C. Guestrin, "Near-Optimal Sensor Placements in Gaussian Processes: Theory, Efficient Algorithms and Empirical Studies," Journal of Machine Learning Research, vol. 9, pp. 235-284, 2008 .

[10] A. Deshpande, S. E. Sarma and V. K Goyal, "Generalized Regular Sampling of Trigonometric Polynomials and Optimal Sensor Arrangement," IEEE Signal Processing Letters, vol. 17, no. 4, pp. 379-382, Apr. 2010.
[11] A. Deshpande and S. E. Sarma, "Error tolerant arrangements of sensors for sampling fields," in 2008 American Control Conference, vol 1-12, pp. 2401-2408, 2008

[12] A. Deshpande, "Coverage problems in mobile sensing," Ph.D Thesis, Massachusetts Institute of Technology, September 2008.

[13] B. Zhang and G. S. Sukhatme, "Adaptive sampling for estimating a scalar field using a robotic boat and a sensor network," in IEEE International Conference on Robotics and Automation, pp. 36733680, 2007.

[14] N. E. Leonard, D. A. Paley, F. Lekien, R. Sepulchre, D. M. Fratantoni, and R. E. Davis, "Collectivemotion, sensor networks, and ocean sampling," in Proceedings of the IEEE, vol. 95, no. 1, pp. 48-74, 2007.

[15] A. Singh, A. Krause and W. Kaiser, "Nonmyopic Adaptive Informative Path Planning for Multiple Robots," in Proc. International Joint Conference on Artificial Intelligence (IJCAI), 2009

[16] A. Singh, A. Krause, C. Guestrin and W. Kaiser, "Efficient Informative Sensing using Multiple Robots," Journal of Artificial Intelligence Research (JAIR), vol. 34, pp. 707-755, 2009

[17] N. K. Yilmaz, C. Evangelinos, P. F. J. Lermusiaux and N. Patrikalakis, "Path Planning of Autonomous Underwater Vehicles for Adaptive Sampling Using Mixed Integer Linear Programming," in IEEE Transactions, Journal of Oceanic Engineering, vol. 33, no. 4, pp. 522-537, 2008.

[18] P. F. J. Lermusiaux, Jr. P. J. Haley and N. K. Yilmaz, "Environmental Prediction, Path Planning and Adaptive Sampling: Sensing and Modeling for Efficient Ocean Monitoring, Management and Pollution Control," Sea Technology, vol. 48, no. 9, pp. 35-38, 2007.

[19] K. Gröchenig, "A Discrete Theory of Irregular Sampling," Linear Algebra and its Applications, vol. 193, pp. 129-150, 1993.

[20] K. Gröchenig, "Reconstruction Algorithms in Irregular Sampling," Mathematics of Computation, vol. 59, pp. 181-194, 1992.

[21] C. E. Shannon, "Communication in the presence of noise," in Proceedings of the IEEE, vol. 86, no. 2, pp. 447-457, 1998.

[22] V. K. Goyal, J. Kovačević and J. A. Kelner, "Quantized frame expansions with erasures," Appl. Comput. Harmon. Anal., vol. 10, no. 3, pp. 203-233, May 2001.

[23] A. Feuer and G. C. Goodwin, "Reconstruction of multidimensional bandlimited signals from nonuniform and generalized samples," IEEE Trans. Signal Process., vol. 53, no. 11, pp. 4273-4282, Nov. 1995.

[24] T. Strohmer, "Computationally attractive reconstruction of bandlimited images from irregular samples," IEEE Trans. Image Process., vol. 6, no. 4, pp. 540-548, Apr. 1997.

[25] A. A. Alonso, C. E. Frouzakis and I. G. Kevrekidis, "Optimal sensor placement for state reconstruction of distributed process systems," AICHE J., vol. 50, pp. 1438-1452, 2004.

[26] A. A. Alonso, I. G. Kevrekidis, J. R. Banga and C. E. Frouzakis, "Optimal sensor location and reduced order observer design for distributed process systems," Comput. Chem. Eng., vol. 28, pp. 27-35, 2004.

[27] Friedrich Pukelsheim, Optimal design of experiments. Philadelphia PA: SIAM, 2006.

[28] D. Ganesan, S. Ratnasamy, H. Wang, and D. Estrin, "Coping with irregular spatio-temporal sampling in sensor networks," SIGCOMM Comput. Commun. Rev., vol. 34, no. 1, pp. 125-130, 2004

[29] G. H. Golub and C. F. Van Loan, Matrix Computations, $3^{\text {rd }}$ ed. Baltimore, MD: Johns Hopkins, 1996, ch. 5.

[30] S.J. Sheather, A modern approach to regression with $R$. New York: Springer, 2009, ch. 4.

[31] K. Gröchenig and T. Strohmer, Theory and Practice of Nonuniform Sampling. Kluwer Academic/Plenum Publishers, 2000, ch. Numerical and theoretical aspects of non-uniform sampling of band-limited images, pp. 283-322.

[32] A. Chatterjee, "An introduction to the proper orthogonal decomposition," Curr. Sci., vol. 78, pp. 808-817, 2000.

[33] J. H. Lienhard IV and J. H. Lienhard V, A heat transfer textbook, $3^{\text {rd }}$ ed. Cambridge, MA: Phlogiston Press, 2008, ch. 11. 